\title{
Quantum Raychaudhuri equation
}

\author{
Saurya Das* \\ Department of Physics and Astronomy, University of Lethbridge, \\ 4401 University Drive, Lethbridge, Alberta T1K 3M4, Canada
}

(Received 3 November 2013; published 23 April 2014)

\begin{abstract}
We compute quantum corrections to the Raychaudhuri equation by replacing classical geodesics with quantal (Bohmian) trajectories and show that they prevent focusing of geodesics and the formation of conjugate points. We discuss implications for the Hawking-Penrose singularity theorems and curvature singularities.
\end{abstract}

DOI: $10.1103 /$ PhysRevD.89.084068

PACS numbers: 04.20.Dw, 03.65.-w, 04.60.-m, 04.62.+v

\section{INTRODUCTION}

The celebrated Hawking-Penrose singularity theorems in general relativity, which show that most reasonable spacetimes are incomplete or singular in a certain precise sense [1], crucially depend on the validity of the Raychaudhuri equation, via the existence of conjugate points that the latter predicts [2]. This equation, although being quite general, nevertheless is completely classical in nature and so are the singularity theorems. However, we know that classical mechanics is an approximation of an underlying quantum world characterized by measurement uncertainties and the absence of particle trajectories. As a result, when the trajectories, or geodesics, and their congruences in the Raychaudhuri equation are replaced with less classical/ more quantum entities, one expects corrections to the equation and a modification of its consequences. In particular, one may hope quantum effects to smooth the sharp focusing of geodesics, the formation of conjugate points and caustics, and ultimately the spacetime singularities.

In this article, we show that by replacing the classical velocity field used in the Raychaudhuri equation, by a quantum velocity field, a first order guiding equation, and the additional quantum potential that comes into play, this focusing is indeed prevented. We discuss its implications for the singularity theorems and curvature singularities. Throughout, we assume a fixed (classical) background spacetime, a four-dimensional differentiable manifold with Lorentzian signature $(+,-,-,-)$.

Starting from a congruence of timelike geodesics (for simplicity, our results easily generalize to null geodesics as well) with tangent vector ("the velocity field") $u^{a}(x)$ parametrized by an affine parameter $\lambda$ along the geodesics, and by $s$ for neighboring geodesics with the deviation vector (or Jacobi field) $\eta^{a}$ (connecting neighboring geodesics), it is straightforward to compute the derivative of $u_{a ; b}$ along a geodesic, as follows:

*saurya.das@uleth.ca

$$
\begin{aligned}
\frac{d u_{a ; b}}{d \lambda} & =u_{a ; b ; c} u^{c}=\left[u_{a ; c ; b}+R_{c b a}{ }^{d} u_{a}\right] u^{c} \\
& =\left(u_{a ; c} u^{c}\right)_{; b}-u_{; b}^{c} u_{a ; c}+R_{c b a}{ }^{d} u^{c} u_{d} \\
& =-u^{c}{ }_{; b} u_{a ; c}+R_{c b a d} u^{c} u^{d} .
\end{aligned}
$$

$R_{a b c d}$ and $R_{a b}$ are the Riemann and Ricci tensors respectively, and we have used the geodesic equation $u_{a ; c} u^{c}=0$, to arrive at the last line. As usual, defining the three-metric $h_{a b}=g_{a b}-u_{a} u_{b}$, and $\theta=h^{a b} u_{a ; b}$ (trace), $\omega_{a b}=u_{[a ; b]}$ (antisymmetric part), $\sigma_{a b}=u_{(a ; b)}-\frac{1}{3} h_{a b} \theta$ (traceless symmetric part) such that $u_{a ; b}=\frac{1}{3} \theta h_{a b}+\sigma_{a b}+\omega_{a b}$, the trace of Eq. (1) yields the Raychaudhuri equation

$$
\frac{d \theta}{d \lambda}=-\frac{1}{3} \theta^{2}-\sigma_{a b} \sigma^{a b}+\omega_{a b} \omega^{a b}-R_{c d} u^{c} u^{d} .
$$

For hypersurface orthogonal geodesics (i.e. $\omega_{a b}=0$ ), and when the strong energy condition via the Einstein equations $R_{c d} u^{c} u^{d}>0$ is satisfied, the rhs of Eq. (2) is negative, and it follows that if the congruence is initially converging $\left[\theta_{0} \equiv \theta(0)<0\right]$, the geodesics will focus, and a caustic will develop within finite value of the affine parameter, $\lambda \leq 3 /\left|\theta_{0}\right|$.

To better illustrate certain points, we first take the nonrelativistic limit of Eq. (2) by replacing $\lambda \rightarrow t$ (the coordinate time), $u^{a}(x) \rightarrow v^{a}(\vec{x}, t),(a=1,2,3)$, $u^{0}=1$, and $R_{c d} u^{c} u^{d} \rightarrow \nabla^{2} V$, where $V(\vec{x}, t)$ is the Newtonian gravitational potential, to obtain ${ }^{1}$

$$
\frac{d \theta}{d t}=-\frac{1}{3} \theta^{2}-\sigma_{a b} \sigma^{a b}+\omega_{a b} \omega^{a b}-\nabla^{2} V .
$$

Using the Poisson equation $\nabla^{2} V=4 \pi G \rho \geq 0$, it is easy to show that analogous focusing of particle trajectories takes place for Eq. (3). Akin to using the geodesic equation to derive Eq. (2), in the above, we used Newton's second law for each particle following the flow of the velocity field $\vec{v}(\vec{x}, t)$,

\footnotetext{
${ }^{1}$ The Raychaudhuri equation in the context of Newtonian gravity has also been discussed in [6].
} 


$$
\frac{d \vec{v}}{d t}=-\vec{\nabla} V(\vec{x}, t)
$$

Next, to obtain quantum corrections to the Raychaudhuri equation, we first note that one now needs a quantum velocity field. This is most easily obtained by writing the wave function of a quantum fluid or condensate as [3]

$$
\psi(\vec{x}, t)=\mathcal{R} e^{i S},
$$

where $\psi(\vec{x}, t)$ is a normalizable wave function, and $\mathcal{R}(\vec{x}, t)$ and $S(\vec{x}, t)$ are real continuous functions [e.g. for a central mass $M$ and test particle mass $m$, these are just the complete set of hydrogen atom wave functions, with negative (bound states) or positive (scattering states) energies, with $e^{2} / 4 \pi \varepsilon_{0} \rightarrow G M m$, and unitary time evolution preserving the normalization of these solutions or a superposition thereof $[4,5]$. One adopts a statistical interpretation with $\rho \equiv|\psi|^{2}$ identified with the density of particles in the fluid (the dynamics guarantees that this relation is preserved in time) and its velocity field as

$$
\vec{v}(\vec{x}, t)=\frac{d \vec{x}}{d t} \equiv \frac{\hbar}{m} \mathcal{I} m\left(\frac{\vec{\nabla} \psi}{\psi}\right)=\frac{\hbar}{m} \vec{\nabla} S(\vec{x}, t) .
$$

Note that this velocity field is irrotational, $\vec{\nabla} \times \vec{v}=0$, i.e. $\omega_{a b}=0$, unless $S$ is singular, signifying the presence of vortices. As usual, one assumes the wave function and consequently $\vec{v}$ as single valued [5]. Substituting in the complex Schrödinger equation yields two real equations

$$
\begin{gathered}
\frac{\partial \rho}{\partial t}+\vec{\nabla} \cdot(\rho \vec{v})=0 \\
m \frac{d \vec{v}}{d t}=-m \vec{\nabla} V+\frac{\hbar^{2}}{2 m} \vec{\nabla}\left(\frac{1}{\mathcal{R}} \nabla^{2} \mathcal{R}\right) .
\end{gathered}
$$

While Eq. (7) is simply the probability conservation law, Eq. (8) resembles the classical Newton's second law of motion but with an extra quantum potential, $V_{Q} \equiv-\frac{\hbar^{2}}{2 m}\left(\frac{1}{\mathcal{R}} \nabla^{2} \mathcal{R}\right)$, in addition to the external (classical) potential $V$, which could be gravitational, for example. Clearly this vanishes in the $\hbar \rightarrow 0$ limit recovering the classical equations of motion and all related classical predictions. Furthermore, although Eqs. (7) and (8) are completely equivalent to the Schrödinger equation, they can be interpreted as giving rise to actual trajectories of particles ("quantal trajectories") initially distributed according to the density $|\psi|^{2}$, and in quantum equilibrium, subject to the external potential $V(\vec{x}, t)$, as well as the additional quantum potential $V_{Q}$. Indeed the latter reproduces the observed interference patterns in a double slit experiment, the Aharonov-Bohm effect, the Stern-Gerlachtype experiments, and all other observed quantum phenomena, and so long as quantum mechanics is valid, no experiments or observations can invalidate the above picture [3]. Thus, we replace classical geodesics with these quantal (Bohmian) trajectories. The Raychaudhuri equation can be rederived for this velocity field but now with the extra potential, i.e. $V \rightarrow V+V_{Q} / m$, resulting in

$\frac{d \theta}{d t}=-\frac{1}{3} \theta^{2}-\sigma_{a b} \sigma^{a b}-\nabla^{2} V+\frac{\hbar^{2}}{2 m^{2}} \nabla^{2}\left(\frac{1}{\mathcal{R}} \nabla^{2} \mathcal{R}\right)$.

Now the expression for the quantum velocity field in Eq. (6), and equivalently, the presence of the quantum potential terms in Eqs. (8) and (9) ensure that the corresponding trajectories do not cross, and there is no focusing for any value of $t$. The easiest way to see this simple yet important result is to note that Eq. (6) is first order in time, and at any time $t$, its right-hand side, and therefore the velocity field are uniquely defined at each point in space. Therefore it gives rise to nonintersecting integral curves or streamlines $[3,7,8]$. In the above, $\psi$ itself evolves according to the Schrödinger equation. Analytical as well as numerical studies indeed demonstrate such interaction between wave packets, between a wave packet and a barrier etc., at short distances, and that although they can come close to each other, they never actually meet or cross [3,7-9]. One may think of this as an effective repulsion between trajectories at short distances, due to the quantum potential. The latter of course vanishes, and the nonrelativistic Raychaudhuri equation (3) is recovered in the $\hbar \rightarrow 0$ limit.

Relativistic generalization follows. We start with a KleinGordon-type equation of the following form, in a fixed classical background, with or without symmetries and with or without or matter,

$$
\left(\square+\frac{m^{2} c^{2}}{\hbar^{2}}-\epsilon_{1} R-\epsilon_{2} \frac{i}{2} f_{c d} \sigma^{c d}\right) \Phi=0,
$$

where the $\epsilon_{1} R, R$ being the curvature scalar term admits of the conformally invariant scalar field equation $\left(\epsilon_{1}=1 / 6\right.$, and $m=0$ ), as well as that obtained from the Dirac equation in curved spacetime $\left(\epsilon_{1}=1 / 4\right)[10,11]$. This term does not contradict observations for ray propagation in curved spacetimes, since normally the $R=0$, the Schwarzschild solution is used. The additional term $-(i / 2) f_{a b} \sigma^{a b}$, where $\sigma^{a b}=(1 / 2)\left[\gamma^{a}, \gamma^{b}\right], \gamma^{a}$ being the Dirac matrices, and $f_{a b}$ an antisymmetric matrix, can also be present in the second order equation derived from the Dirac equation in curved spacetimes $\left(\epsilon_{2}=1\right.$ for fermions and 0 for bosons) [11]. Once again, the wave function $\Phi$ is nomalizable and single valued, as required for a quantum description of the system (again, for example for some of the well-studied spacetimes with curvature singularities, such as the Schwarzschild and Reissner-Nordström metrics, these could be the wave functions in $[[12,13]]$ or the ones used in [14] in the context of Bohmian trajectories) and expressible as in Eq. (5). Note that here one has $\Phi$ on a 
fixed (nondynamical) background spacetime, and not part of a coupled Einstein-scalar field system, in which both the field and the metric are dynamical. Now the 4-momentum, four-velocity field and "coordinate velocity" are defined respectively as [15-17]

$$
\begin{gathered}
k_{a}=\partial_{a} S, \\
u_{a}=c \frac{d x_{a}}{d \lambda}=\frac{\hbar k_{a}}{m}, \\
\vec{v}=\frac{d \vec{x}}{d t}=-c^{2} \frac{\vec{\nabla} S}{\partial^{0} S} .
\end{gathered}
$$

As before, one may replace the classical relativistic velocity field with the above, which would correctly predict all observations. Substituting Eq. (5) in Eq. (10) now yields the two equations

$$
\begin{gathered}
\partial^{a}\left(\mathcal{R}^{2} \partial_{a} S\right)=\frac{\epsilon_{2}}{2} f_{c d} \sigma^{c d} \mathcal{R}^{2}, \\
k^{2}=\frac{(m c)^{2}}{\hbar^{2}}-\epsilon_{1} R+\frac{\square \mathcal{R}}{\mathcal{R}},
\end{gathered}
$$

where again, Eq. (14) is the conservation equation, while Eq. (15) yields the modified geodesic equation with the relativistic quantum potential term $V_{Q}=\frac{\hbar^{2}}{m^{2}} \frac{\square \mathcal{R}}{\mathcal{R}}$,

$$
u_{; a}^{b} u^{a}=-\frac{\epsilon_{1} \hbar^{2}}{m^{2}} R^{; b}+\frac{\hbar^{2}}{m^{2}}\left(\frac{\square \mathcal{R}}{\mathcal{R}}\right)^{; b} .
$$

Then the quantum corrected Raychaudhuri equation takes the form

$$
\begin{aligned}
\frac{d \theta}{d \lambda}= & -\frac{1}{3} \theta^{2}-\sigma_{a b} \sigma^{a b}-R_{c d} u^{c} u^{d} \\
& -\frac{\epsilon_{1} \hbar^{2}}{m^{2}} h^{a b} R_{; a ; b}-\frac{\hbar^{2}}{m^{2}} h^{a b}\left(\frac{\square \mathcal{R}}{\mathcal{R}}\right)_{; a ; b} .
\end{aligned}
$$

In this case too, the first order equation (13), the uniqueness of the velocity field at each point in space, and the quantum potential in Eqs. (16) and (17) ensure that the trajectories (geodesics) do not cross, again resulting in no focusing and no conjugate points for any finite value of the affine parameter. Again, it can be seen that the quantum potential vanishes, and the classical Raychaudhuri equation (2) is recovered in the $\hbar \rightarrow 0$ limit. Generalization to null geodesics and to Maxwell fields is straightforward (the $m$ will not enter when these equations are derived for null geodesics from first principles) [15]. Note that the exact form of the wave equation and its various modifications are not important for the argument. All that one needs to assume is the existence of such a theory, and the first order equations of the form (13), and the no-crossing result continues to hold.

\section{IMPLICATIONS FOR SINGULARITY THEOREMS}

Although the unboundedness of curvature scalars is often regarded as a signature of singular spacetimes, this is neither necessary (e.g. removing a wedge from Minkowski space makes it singular) nor sufficient (e.g. when they are reachable only in infinite proper time, or the difficulties in specifying singularity as a "place" for generic spacetimes). Therefore one equates the incompleteness of geodesics (which is easier to determine), equivalently the termination of existence of a particle (or photon), to singular or pathological spacetimes $[1,18]$. It can be shown that the focusing of geodesics implies the existence of pairs of conjugate points, where $\vec{\eta}$ vanishes for neighboring geodesics, which in turn implies that sufficiently long geodesics cannot be maximal length curves. The existence of maximal geodesics is predicted on the other hand by a set of global arguments for globally hyperbolic spacetimes. This apparent contradiction is resolved by requiring that sufficiently long geodesics cannot exist, leading to geodesic incompleteness and "singular spacetimes," which is the essence of the singularity theorems (as mentioned earlier, throughout this article, we omit the finer distinction between timelike and null geodesics, since we expect our results to hold for either) [18]. However, we know that in the quantum picture, particles do not follow classical trajectories or geodesics; therefore the Hawking-Penrose singularity theorems, although still valid, lose much of their original motivation, and therefore need to be replaced by a quantum version. As we have shown here, particles can be thought of as following quantal (Bohmian) trajectories instead (as they correctly predict all observations); therefore these are natural candidates for replacing geodesics in the singularity theorems. However, since these are complete (i.e. do not end) and do not have conjugate points (i.e. $\vec{\eta}$ never vanishes), the resultant "semiclassical" version of the singularity theorems now do not predict the existence of singularities. Furthermore, as shown below, regions of unbounded curvature are never reached by the quantal trajectories. Therefore, either one would have to find another way to characterize singularities using quantum mechanics, and applicable to a wide class of spacetimes, or would have to conclude that singularities are in fact avoidable.

\section{IMPLICATIONS FOR CURVATURE SINGULARITIES}

Next, consider the geodesic deviation equation modified by the quantum potential term (we omit the $\varepsilon_{1}$ term here) 
$\frac{D^{2} \eta^{a}}{d \lambda^{2}}=-\frac{1}{c^{2}} R_{b f c}^{a} u^{b} u^{c} \eta^{f}-\frac{\hbar^{2}}{m^{2} c^{2}}\left[\left(\frac{\square \mathcal{R}}{\mathcal{R}}\right)^{; a}\right]_{; c} \eta^{c}$.

For spacetimes in which curvature scalars (such as the Kretschmann scalar $R_{a b c d} R^{a b c d}$ ) blow up (e.g. at $r=0$ for certain black holes), the deviation vector $\vec{\eta} \rightarrow 0$. Since the quantum Raychaudhuri equation, as well as the additional term in Eq. (18) above show on the other hand that $\vec{\eta} \neq 0$ at all times, these extreme curvature regions are not accessible, and observed curvature components and scalars would also remain finite (albeit large) at all times.

To summarize, we have shown that replacing classical trajectories or geodesics by their quantum counterparts gives corrections to the Raychaudhuri equation, which naturally prevents focusing and the formation of conjugate points. Therefore, if one replaces classical geodesics with quantal (Bohmian) trajectories in the singularity theorems, then the quantum version of these theorems do not show that spacetime singularities are inevitable. We reiterate that we have simply rewritten regular quantum mechanics in a convenient form, in which the no crossing of trajectories due to the first order evolution equation becomes transparent. Another way of looking at this is that the quantum potential, although being small, causes deviations from classical trajectories at short distances sufficient for trajectories to not cross each other. Also, we have not assumed spherical or any other symmetry in our analysis, and our results are valid for all spacetimes. Our results hold for bosons as well as fermions (note that we have included the Dirac equation), although for fermions, one might encounter additional exchange forces at small distances, further inhibiting the focusing of geodesics. To our knowledge, this is the first time that systematic quantum corrections to the Raychaudhuri equation have been computed and its implications examined, without using any specific formulation of quantum gravity or invoking special symmetries. It is tempting to speculate that for curved spacetimes, the quantum potential becomes important, and the no convergence would be seen near the Planck length, the latter being the natural scale in quantum gravity. It would be interesting to investigate the fate of these quantum trajectories for values of the affine parameter near or exceeding $3 /\left|\theta_{0}\right|$. A combination of analytical and numerical studies should shed more light on these issues. Finally, it may be argued that our assumption of a smooth background manifold may break down at small scales, and especially in regions of high curvatures being replaced by a more fundamental "quantum structure." This is certainly a possibility, although perhaps not compelling. Furthermore, as remarked earlier, and as our Eq. (18) suggests, regions of very high curvatures may in fact be inaccessible.

\section{ACKNOWLEDGMENTS}

I thank S. Braunstein, R. K. Bhaduri, A. Figalli, D. Hobill, S. Kar, G. Kunstatter, R. B. Mann, R. Parwani, T. Sarkar, L. Smolin and R. Sorkin for discussions and correspondence, and also the anonymous referees for useful suggestions which helped improve the manuscript, and the IQST and PIMS, University of Calgary, for hospitality, where part of this work was done. I also thank the Perimeter Institute for Theoretical Physics for hospitality through their affiliate program. This work is supported by the Natural Sciences and Engineering Research Council of Canada.
[1] R. Penrose, Phys. Rev. Lett. 14, 57 (1965); S. W. Hawking and R. Penrose, Proc. R. Soc. A 314, 529 (1970).

[2] A. K. Raychaudhuri, Phys. Rev. 98, 1123 (1955).

[3] D. Bohm, Phys. Rev. 85, 166 (1952); D. Bohm, B. J. Hiley, and P. N. Kaloyerou, Phys. Rep. 144, 321 (1987).

[4] C. Cohen-Tannoudji, B. Dio, and F. Laloë, Quantum Mechanics (Hermann, Paris, 1977), Vol. 1, Chap. VII.

[5] P. R. Holland, The Quantum Theory of Motion (Cambridge University Press, Cambridge, England, 1993), Chap. 3.

[6] J. O. Weatherall, arXiv:1308.1722.

[7] D. A. Deckert, D. Dürr, and P. Pickl, J. Phys. Chem. A 111, 10325 (2007); A. S. Sanz, J. Phys. Conf. Ser. 361, 012016 (2012).

[8] A. Figalli, C. Klein, P. Markowich, and C. Sparber, arXiv:1202.3134.

[9] X. Oriols, F. Martin, and J. Suñe, Phys. Rev. A 54, 2594 (1996).

[10] M. D. Pollock, Acta Phys. Pol. B 41, 1827 (2010).
[11] E. Schrödinger, Sitzungsber. Preuss. Akad. Wiss. Phys. Math. Kl. 105 (1932).

[12] S. W. Hawking, Commun. Math. Phys. 43, 199 (1975).

[13] K. Melnikov and M. Weinstein, Int. J. Mod. Phys. D 13, 1595 (2004).

[14] R. Tumulka, arXiv:0708.0070; Gen. Relativ. Gravit. 42, 303 (2010).

[15] M. Sasaki, Prog. Theor. Phys. 90, 753 (1993).

[16] G. Horton, C. Dewdney, and A. Nesteruk, J. Phys. A 33, 7337 (2000); G. Horton, C. Dewdney, and U. Neeman, arXiv:quant-ph/0109059.

[17] D. Dürr, S. Goldstein, T. Norsen, W. Struyve, and N. Zanghi, arXiv:1307.1714; H. Nikolić, J. Phys. Conf. Ser. 67, 012035 (2007).

[18] See, e.g., R. M. Wald, General Relativity (University of Chicago Press, Chicago, 1984), Chap. 9, or S. W. Hawking and G. F. R. Ellis, The Large Scale Structure of Spacetime (Cambridge University Press, Cambridge, England, 1973), Chap. 8. 